ISSN 2076-393X

www.mdpi.com/journal/vaccines

Article

\title{
EBV-Associated Cancer and Autoimmunity: Searching for Therapies
}

\section{Giovanni Capone ${ }^{\mathbf{1}}$, Candida Fasano ${ }^{1}$, Guglielmo Lucchese ${ }^{2}$, Michele Calabrò ${ }^{1}$ and Darja Kanduc ${ }^{1, *}$}

1 Department of Biosciences, Biotechnologies and Biopharmaceutics, University of Bari, Bari 70126, Italy; E-Mails: g.capone@ biologia.uniba.it (G.C.); candida.fasano@gmail.com (C.F.); michele.calabro@hotmail.it (M.C.)

2 Brain and Language Laboratory, Free University of Berlin, 14195 Berlin, Germany; E-Mail: guglielmo.lucchese@ @otmail.com

* Author to whom correspondence should be addressed; E-Mails: dkanduc@ gmail.com; darja.kanduc@uniba.it; Tel.: +39-80-544-3321; Fax: +39-80-544-3317.

Academic Editor: Lenora (Nora) Disis

Received: 25 September 2014 / Accepted: 27 January 2015 / Published: 5 February 2015

\begin{abstract}
Epstein-Barr virus (EBV) infects B-, T-, and NK cells and has been associated not only with a wide range of lymphoid malignancies but also with autoimmune diseases such as lupus erythematosus, rheumatoid arthritis and, in particular, multiple sclerosis. Hence, effective immunotherapeutic approaches to eradicate EBV infection might overthrow cancer and autoimmunity incidence. However, currently no effective anti-EBV immunotherapy is available. Here we use the concept that protein immunogenicity is allocated in rare peptide sequences and search the Epstein-Barr nuclear antigen 1 (EBNA1) sequence for peptides unique to the viral protein and absent in the human host. We report on a set of unique EBV EBNA1 peptides that might be used in designing peptide-based therapies able to specifically hitting the virus or neutralizing pathogenic autoantibodies.
\end{abstract}

Keywords: EBV EBNA1; cancer; autoimmunity; peptide matching; low-similarity peptides; anti-EBV vaccine; peptide-therapy 


\section{Introduction}

Epstein-Barr virus (EBV) is implicated in the development of a wide range of lymphoproliferative disorders, including Burkitt's lymphoma [1], classic Hodgkin's lymphoma (HL) [2], non-Hodgkin lymphoma (NHL) [3,4], nasal NK-cell lymphoma [5,6] and, in addition, in nasopharyngeal carcinoma and a subset of gastric cancers [7-9].

Moreover, autoimmune diseases may follow EBV-infection. In fact, a potential role of EBV in systemic lupus erythematosus (SLE) has been suggested [10], and development of lupus-like autoantibodies (AAbs) following immunization with Epstein-Barr nuclear antigen 1 (EBNA1) fragments has been reported in animals [11]; links between rheumatoid arthritis (RA) and EBV have been reported [12,13]; also, association between anti-EBNA titers and risk of multiple sclerosis (MS) has been described [14] and oligoclonal bands immunoreactive with EBV EBNA1 have been found in patients with MS [15].

On the whole, given the fact that EBV has been detected in all populations and geographical areas [16] and that the virus has an efficacious immune escape strategy [17], EBV-infection may reasonably be considered as contributing to the continuously increasing incidence of cancer and autoimmunity worldwide. Indeed, for example, HL and NHL have been ranked respectively as the 25th and 10th most common cancers worldwide in 2012 [18].

Likewise, MS cases increased in the period 2008-2013 (from 2.1 to 2.3 million) [19]; the incidence of SLE is nearly tripled in the last 40 years of the 20th century [20], and RA affects approximately $1 \%$ of the worldwide population [21]. Hence, anti-EBV immunotherapies might overthrow the incidence of highly common cancers such as lymphomas as well as the increasing incidence of autoimmune diseases such as SLE and MS. However, notwithstanding the need of fighting EBV infection, currently effective vaccines are not yet available.

Here, we use the concept that immunogenic properties are allocated in rare peptide modules along a protein sequence [22-25] and search the EBV EBNA1 sequence for peptides unique to the viral protein and absent in the human host. We find and describe a set of unique EBV EBNA1 peptides that might be used in designing peptide-based vaccines able to specifically hit the viral protein without crossreacting with the host proteins. The present data appear of special interest since might lead to vaccination protocols for a global EBV eradication. Moreover, such unique EBV epitopic peptides might find application to specifically neutralize pathogenic AAbs associated with the autoimmune diseases that have been related to EBV infection.

\section{Experimental Section}

Analyses were conducted on the primary amino acid (aa) sequence of EBV EBNA1 protein (UniProtKB/Swiss-Prot ID: Q3KSS4, 641 aa) from EBV strain GD1, GenBank: AY961628.3 (http://www.ebi.ac.uk/ena/data/view/AY961628) [26]. As a control, EBV GP350 protein (UniProtKB/Swiss-Prot ID: Q3KST4, 856 aa) was also analyzed.

Sequence similarity analysis of the EBNA1 protein $v s$. the human proteome was performed using pentapeptides as scanning probes. The viral protein was dissected into 5-mers sequentially overlapping by four residues (i.e., MSDEG, SDEGP, DEGPG, EGPGT, etc.). For each viral 5-mer, the human 
proteome was searched for instances of the same identical 5-mer using PIR match program (pir.georgetown.edu/pirwww/search/peptide.shtml) [27]. Any such occurrence was termed a match.

The cross-reactivity potential for each pentapeptide sharing was evaluated using Immune Epitope Database and Analysis Resources (IEDB; http://www.iedb.org/) [28] to search for EBNA1-derived B- and/or T-cell epitopes that had been experimentally validated as immunopositive in the human host.

Consensus peptide sequences were defined by ClustalW multialignment analysis (http://www.uniprot.org/align/) [29] of three EBV EBNA1 sequences corresponding to: Q3KSS4 (from GD1 strain, NCBI Tax ID: 10376); P03211 (from B95-8 strain, NCBI Tax ID: 10377); and Q1HVF7 (from AG876 strain, NCBI Tax ID: 82830).

\section{Results}

\subsection{Peptide Commonality between EBNA1 and the Human Proteome}

As exhaustively discussed by Benjamin et al. [30], immune "determinants are conformational in the sense that the antibody combining sites will bind with a measurable affinity only to that population of antigen conformers which presents a complementary constellation of interacting side chains. It follows that antigenic determinants are topographic, i.e., composed of structures on the protein surface. Topographic determinants may be contained within a single segment of the amino acid sequence (but not necessarily involving all contiguous residues in the segment), or assembled from residues far apart in the amino acid sequence but brought together on the surface by the folding of the protein antigen" [30].

By considering that conformational and sequential determinants do not imply different antigenic binding mechanisms [30], and that: (1) specific Abs can be raised against peptides of undefined conformation [31]; (2) short synthetic peptides capable of eliciting protein-reactive sera are frequently represented in the primary sequence of a protein [32]; (3) such immunogenic peptides are frequently represented in the primary sequence of a protein [33]; and (4) generation of protein-reactive Abs by short peptides is an event of high frequency [34], research on antigen epitopes has been mainly directed toward linear aa sequences as documented by epitope databases such as IEDB [28], Tri-peptide similarity and Propensity scores (SVMTriP) [35], Linear Epitope Prediction by Propensities and Support Vector Machine (LEPS) [36], and Linear B-cell Epitope Prediction Server (LBtope) [37].

Here, analyses focused on short determinants formed by sequential contiguous aa and used a measurement unit defined by peptide length (i.e., the pentapeptide) for peptide uniqueness comparisons. In fact, a number of reports have reiterated the concept that a grouping of 5 aa residues can be immunogenic [38-44] and have immunorecognition capability [44-48]. Hence, a pentapeptide may represent an appropriate length unit to be used for analyzing the potential for immune crossreactivity of peptide sharing among proteins $[24,49,50]$.

EBV EBNA1 antigen was chosen since it is constitutively expressed in EBV malignancies. In fact, it is often the only latent EBV antigen expressed in Burkitt's lymphoma and nasopharyngeal carcinoma [51]; it appears to be involved in the development of gastric cancer [52]. Moreover EBNA1 represents a prime target for T-cell-based immunotherapy [53-55]. 
Figure 1 illustrates the similarity profile of EBV EBNA1 protein sequence to the human proteome at the pentapeptide level. It can be seen that the most part of the 637 pentapeptides that sequentially form the viral protein, are repeatedly present in human proteins. Namely, 622 out of 637 viral pentapeptides are present 66,052 times (including multiple occurrences) in 7312 human proteins [56].

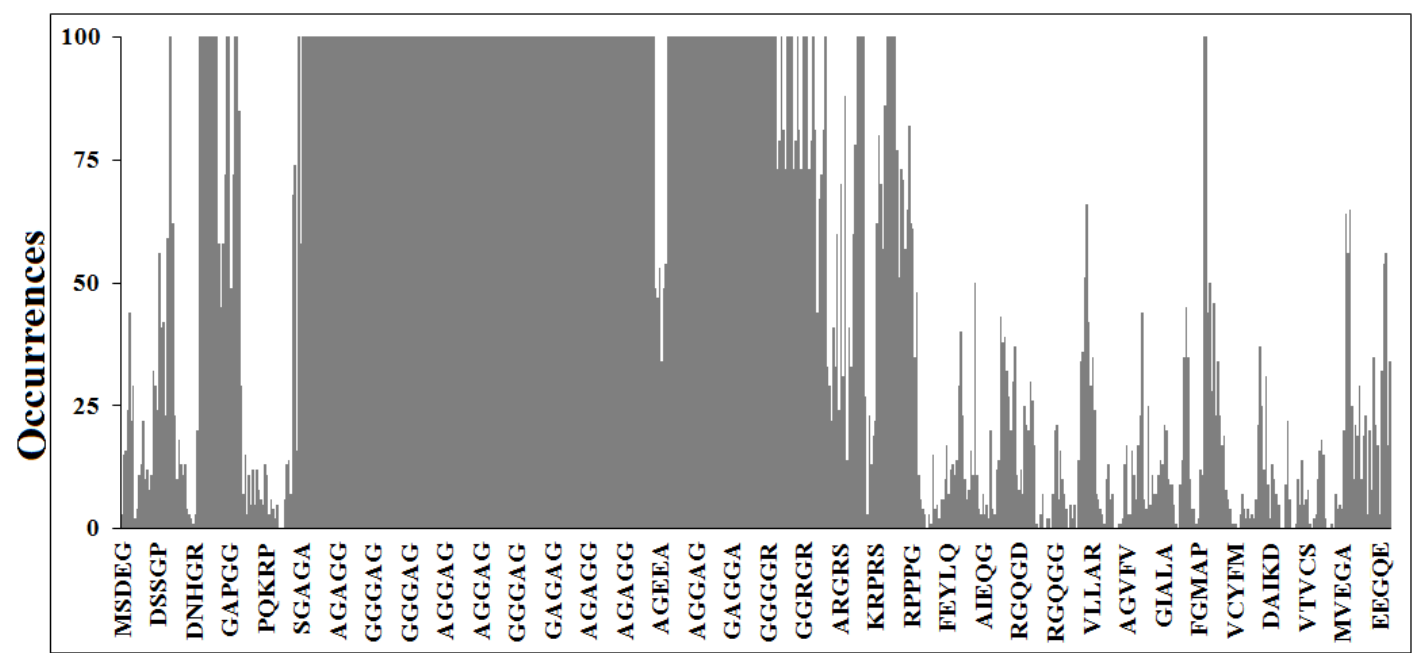

EBV EBNA1 pentapeptides

Figure 1. Pentapeptide identity platform shared between Epstein-Barr virus (EBV) Epstein-Barr nuclear antigen 1 (EBNA1) protein and the human proteome. Peptide aa sequences in one letter code.

Qualitatively, analysis of the pentapeptide overlap using PIR database highlights that (1) human proteins playing crucial roles in cell functions are involved in the peptide sharing and, in addition; (2) in many instances, many consecutively overlapping pentapeptides forming long peptide stretches are shared. For example:

(1) The EBNA154-62PGAPGGSGS nonapeptide is common to the human protein zyxin (UniProtKB/Swiss-Prot IDs: Q15942, ZYX_HUMAN), a component of a signal transduction pathway that mediates adhesion-stimulated changes in gene expression [57];

(2) The AGAGGAGAG nonapeptide is repeated five times in EBNA1, and is shared with the human ubiquitin-conjugating enzyme E2 Q1 (UniProtKB/Swiss-Prot IDs: Q7Z7E8, UB2Q1_HUMAN) that catalyzes the covalent attachment of ubiquitin to other proteins [58];

(3) The GGGAGGAGG nonapeptide is repeated five times in EBNA1, and is shared with the transcription factor jun-B (UniProtKB/Swiss-Prot IDs: P17275, JUNB_HUMAN). JUNB is a transcription factor involved in regulating gene activity following the primary growth factor response [59] and inhibits proliferation and transformation in B-lymphoid cells [60];

(4) In addition, the GGGAGGAGG nonapeptide is also present in the human far upstream element-binding protein 2 (UniProtKB/Swiss-Prot IDs: Q92945, FUBP2_HUMAN). FUBP2 binds to the dendritic targeting element and may play a role in mRNA trafficking. FUBP2 also mediates exon inclusion in transcripts that are subject to tissue-specific alternative splicing [61];

(5) The EBNA140-50GRGRGRGRGRGRGRG undecapeptide is also present in the human small nuclear ribonucleoprotein SmD1, a core component of the spliceosomal U1, U2, U4 and U5 small 
nuclear ribonucleoproteins (snRNPs), the building blocks of the spliceosome [62]. Importantly, this dipeptide Gly-Arg repeat crossreacts with Abs against an SmD-like epitope recognized by sera from SLE patients [63], thus possibly underlying the EBV-SLE association [10,11].

Moreover, it has to be underlined that the above listed peptide identities occur along the central 200 aa long Gly-Ala repeat of EBNA1, a viral region that has immunosuppressive properties since it may affect MHC I restricted responses by inhibiting antigen processing via the ubiquitin/proteasome pathway $[64,65]$.

\subsection{Searching for a Vaccine: Unique EBNA1 Sequences}

Translating the data exposed above to the context of the present study, Figure 1 illustrates the concept that using vaccines based on the entire EBNA1 antigen might potentially lead to a plethora of crossreactions with proteins exerting fundamental roles in the human host. On the contrary [22-25], only pentapeptides unique to EBNA1 might lead to peptide-based vaccines exempt of crossreaction. The 15 viral pentapeptides absent in the human proteome are illustrated in Table 1.

Table 1. Peptide profile of EBV EBNA1 protein primary sequence vs. the human proteome: the unique EBNA1 identity spots at the pentapeptide level.

\begin{tabular}{cccccc}
\hline Position $^{\text {a }}$ & Sequence $^{\text {b,c }}$ & Position $^{\text {a }}$ & Sequence $^{\text {b,c }}$ & Position $^{\text {a }}$ & Sequence $^{\text {b,c }}$ \\
\hline $80-84$ & IGCKG & $467-471$ & KHRGQ & $584-588$ & MTKPA \\
$81-85$ & GCKGA & $476-480$ & PKFEN & $588-592$ & APTCN \\
$82-86$ & CKGAH & $499-503$ & EEGNW & $589-593$ & PTCNI \\
$461-465$ & KGGWF & $500-504$ & EGNWV & $598-602$ & CSFDD \\
$464-468$ & WFGKH & $561-565$ & YFMVF & $609-613$ & WFPPM \\
\hline
\end{tabular}

The EBV EBNA1 pentapeptides with zero similarity to the human proteome (e.g., viral pentapeptides absent in the human proteins) are sequentially listed by aa position along the viral protein. ${ }^{\text {a }}$ Aa position along the viral protein; ${ }^{\mathrm{b}}$ Aa sequences given in one-letter code; ${ }^{\mathrm{c}}$ Consecutively overlapping pentapeptides given in bold.

The pentapeptides described in Table 1 might effectively be a basis for efficacious anti-EBNA1 vaccines also in light of the fact that such unique viral peptide sequences are part of EBNA1 epitopes already experimentally validated as immunopositive in the human host. Indeed, as shown in Table 2, 26 EBNA1-derived epitopes cataloged at IEDB and validated as immunopositive in humans host 13 out of the 15 unique viral pentapeptides described in Table 1.

Sequence comparison analysis also shows that 6 out of the 15 pentapeptides unique to EBNA1 from EBV strain GD1 are also conserved in EBNA1 from B95-8 and AG876 strains (see Table 3). This means that using peptide sequences described in Table 3 might offer the possibility of hitting different EBV strains using a single vaccine preparation. 
Table 2. Thirteen out of the 15 pentapeptides unique to EBV EBNA1 and absent in the human proteome, are distributed among 26 EBV EBNA1-derived epitopes are immunoreactive in humoral and/or cellular immunoassays.

\begin{tabular}{cccc}
\hline IEDB ID $^{\text {a }}$ & Epitope Sequence $^{\text {b,c }}$ & Immune Context & References \\
\hline 1219 & aevlkdaikdlvMTKPAptc & $\mathrm{B}$ & {$[66]$} \\
8395 & dggrrkKGGWFGKHr & $\mathrm{T}$ & {$[67,68]$} \\
8397 & dggrrkKGGWFgrhr & $\mathrm{T}$ & {$[69]$} \\
11651 & EEGNWVagvfvyggsktslynlrrg & $\mathrm{T}$ & {$[53]$} \\
26761 & ikdlvMTKPAPTCNI & $\mathrm{T}$ & {$[70]$} \\
30951 & KGGWFGKHRGQggs & $\mathrm{B}, \mathrm{T}$ & {$[71,72]$} \\
39079 & lresivcYFMVFlqthifae & $\mathrm{T}$ & {$[67]$} \\
39080 & lresivcYFMVFlqthifaevlkda & $\mathrm{T}$ & {$[53]$} \\
45378 & nPKFENiaeglrall & $\mathrm{T}$ & {$[67-69]$} \\
45379 & nPKFENiaeglrallarshv & $\mathrm{T}$ & {$[55,73]$} \\
45380 & nPKFENiaeglrallarshverttde & $\mathrm{T}$ & {$[74,75]$} \\
48948 & ppWFPPMvegaaa & $\mathrm{T}$ & {$[76]$} \\
49056 & pqpgplresivcYFMVFlqt & $\mathrm{T}$ & {$[53]$} \\
49593 & PTCNIkatvCSFDDgvdlpp & $\mathrm{T}$ & {$[67,69]$} \\
49594 & PTCNIkvtvCSFDDgvdlppWFPPM & $\mathrm{T}$ & {$[53]$} \\
55299 & rpqkrpscIGCKGthggtga & $\mathrm{B}$ & {$[66]$} \\
55336 & rpscIGCKGthggtg & $\mathrm{T}$ & {$[77]$} \\
55684 & rrpqkrpscIGCKGt & $\mathrm{T}$ & {$[67,69]$} \\
56433 & rvtvCSFDDgvdlppWFPPM & $\mathrm{T}$ & {$[67]$} \\
59875 & snPKFENiaeglrvllarsh & $\mathrm{T}$ & {$[54,55]$} \\
67891 & vcYFMVFlqthifae & $\mathrm{T}$ & {$[70]$} \\
69559 & vlkdaikdlvMTKPAPTCNI & $\mathrm{T}$ & {$[67,69]$} \\
73861 & YFMVFlqthifae & $\mathrm{T}$ & {$[76]$} \\
73862 & YFMVFlqthifaevl & $\mathrm{T}$ & {$[77]$} \\
93570 & PKFENiaeglr & $\mathrm{T}$ & {$[78]$} \\
118828 & gsgprhrdgvrrpqkrpscIGCKGthggtg & $\mathrm{B}$ & {$[79]$} \\
\hline
\end{tabular}

${ }^{a}$ EBV EBNA1-derived epitopes are listed according to increasing IEDB ID number. For further details and reference(s) see IEDB [28]; ${ }^{\mathrm{b}}$ Only EBV EBNA1-derived epitopes that had been experimentally validated as immunopositive in the human host are reported; ${ }^{\mathrm{c}}$ In each epitope, EBV EBNA1 pentapeptide(s) absent in the human proteome are given in capital.

Table 3. Conservation of EBNA1 unique peptide regions among EBV GD1, B95-8, and AG876 strains.

\begin{tabular}{ccccccc}
\hline EBV Strain & ID & \multicolumn{5}{c}{ Consensus Peptide Sequences } \\
\hline GD1 & Q3KSS4 & IGCKG & GKHRG & APTCNI & CSFDD & WFPPM \\
B95-8 & P03211 & IGCKG & GKHRG & APTCNI & CSFDD & WFPPM \\
AG876 & Q1HVF7 & IGCKG & GKHRG & APTCNI & CSFDD & WFPPM \\
\hline
\end{tabular}

EBNA1 sequences were aligned using ClustalW program (http://www.uniprot.org/align/) [29]. The analyzed sequences are reported by SwissProt/UniProtKB ID. EBV strains are described at www.uniprot.org. 


\section{Discussion}

Numerous immunological approaches have been explored to fight EBV. A few examples are:

- EBNA1 targeting to dendritic cells to stimulate protective T-cell responses [80];

- EBV-specific cytotoxic T-lymphocytes to control EBV-related lymphoproliferation [81];

- a live recombinant virus, expressing under the $11 \mathrm{~K}$ vaccinia promoter the major EBV membrane antigen BNLF-1 MA (GP 220-340) to protect against and/or delay EBV infection [82];

- GP350(1-470)-based vaccines in order to prevent the virus binding to CD21 on B-cells [83-85];

- EBV vaccines based on virus-like particles that mimic the structure of the parental virus but lack the viral genome [86];

- adoptive transfer of EBV specific $\mathrm{CD}^{+} \mathrm{T}$ cell clones $[87,88]$.

However, in spite of the numerous and intensive studies, currently there is no specific treatment/vaccine against EBV infection [89].

The present study proposes the principle of peptide uniqueness [22-25] to construct and develop specific and efficacious EBV vaccines that are exempt from potential crossreactions. In this regard, our findings might also help avoid potential crossreactions in EBV GP350 antigen-based vaccine currently under trial to prevent infectious mononucleosis $[83,84]$. As a matter of fact, the pentapeptide identity platform shared by EBV GP350 antigen and the human proteome (Appendix Figure A1) reproposes a relevant pentapeptide overlap between EBV GP350 and human proteins, in analogy to the results obtained for EBV EBNA1 and illustrated in Figure 1.

A lack of crossreactivity acquires a clinical importance also in light of the fact that autoimmunity has been associated to high anti-EBV immune responses in the human host. Indeed, increased anti-EBV EBNA1 immune responses predict conversion to MS [90,91], and, likewise, high immune responses to EBV have been found in individuals with systemic and organ specific autoimmune disorders such as RA and SLE [92]. In particular, it is of special relevance to the present study that SLE patients are characterized by a heterogeneous immune response to a dipeptide repeat GR (Gly-Arg). As reported above, such a dipeptide repeat GR represents a shared sequence between EBNA1 and SmD1 and is also a well characterized epitope (IEDB ID: 117518) [64,93].

Such an approach would have the added advantage of preventing crossreactions that appear to be at the basis of the autoimmune diseases presumably associated with immune responses that follow EBV infection. Given the theoretically highest safety of vaccines based on peptides unique to infectious pathogens, intensive prophylactic campaigns of anti-EBV vaccination might be possible, thus promising a global eradication of EBV in the human population.

Therapeutically, our findings open the way to verifying the possibility of using unique EBV epitopic peptides to treat autoimmune diseases related to EBV infection. In fact, short peptides are too small to stimulate antigenic responses to pathogenic regions of autoantigens and may represent effective tolerogens capable of anergizing autoreactive T cells [94,95]. Therefore, unique EBV epitopic peptides might be used to selectively block and neutralize circulating autoreactive AAbs in EBV-associated autoimmune diseases. In this regard, we already used the concept of peptide uniqueness to search Pemphigus vulgaris (PV) autoantigen desmoglein-3 (Dsg3) for peptide sequence(s) to be used for blocking autoreactive AAbs [96,97]. The search led to Dsg349-60REWVKFAKPCRE peptide sequence 
that (1) is uniquely expressed in Dsg3 and, consequently, cannot evoke collateral secondary autoimmune cross-reactions; (2) is allocated in a Dsg3 domain involved in the intramolecular epitope spreading characterizing the progression of PV from mucous to muco-cutaneous stage [98]; (3) did not produce pathogenic Abs in an animal model [99]. Remarkably, topical administration of the Dsg349-60REWVKFAKPCRE peptide was able to stably reverse a terminal stage of PV complicated by diabetes and cataract disease [100].

\section{Conclusions}

The present study applies the concept of peptide uniqueness to develop new therapeutic approaches against EBV infection and the associated cancer and immune pathologies. The data warrant further studies and research since treatments based on peptides uniquely owned by EBV would offer high specificity as well as the advantage of a lack of adverse events in the host.

\section{Acknowledgments}

Giovanni Capone and Candida Fasano are volunteer researchers at the University of Bari. Guglielmo Lucchese is supported by Deutscher Akademischer Austauschdienst (DAAD). Michele Calabrò is a student of the course "Analytical Morphometry and Models of Molecular Medicine". Darja Kanduc is funded by the Ministry of University and Research of Italy.

\section{Author Contributions}

Giovanni Capone, Candida Fasano, Guglielmo Lucchese and Michele Calabrò performed the computational analyses; Giovanni Capone also contributed to the project definition. Darja Kanduc proposed the original idea, supervised the work, interpreted the data and wrote the paper. All authors discussed and approved the paper.

\section{Conflicts of Interest}

The authors declare no conflict of interest. 


\section{Appendix}

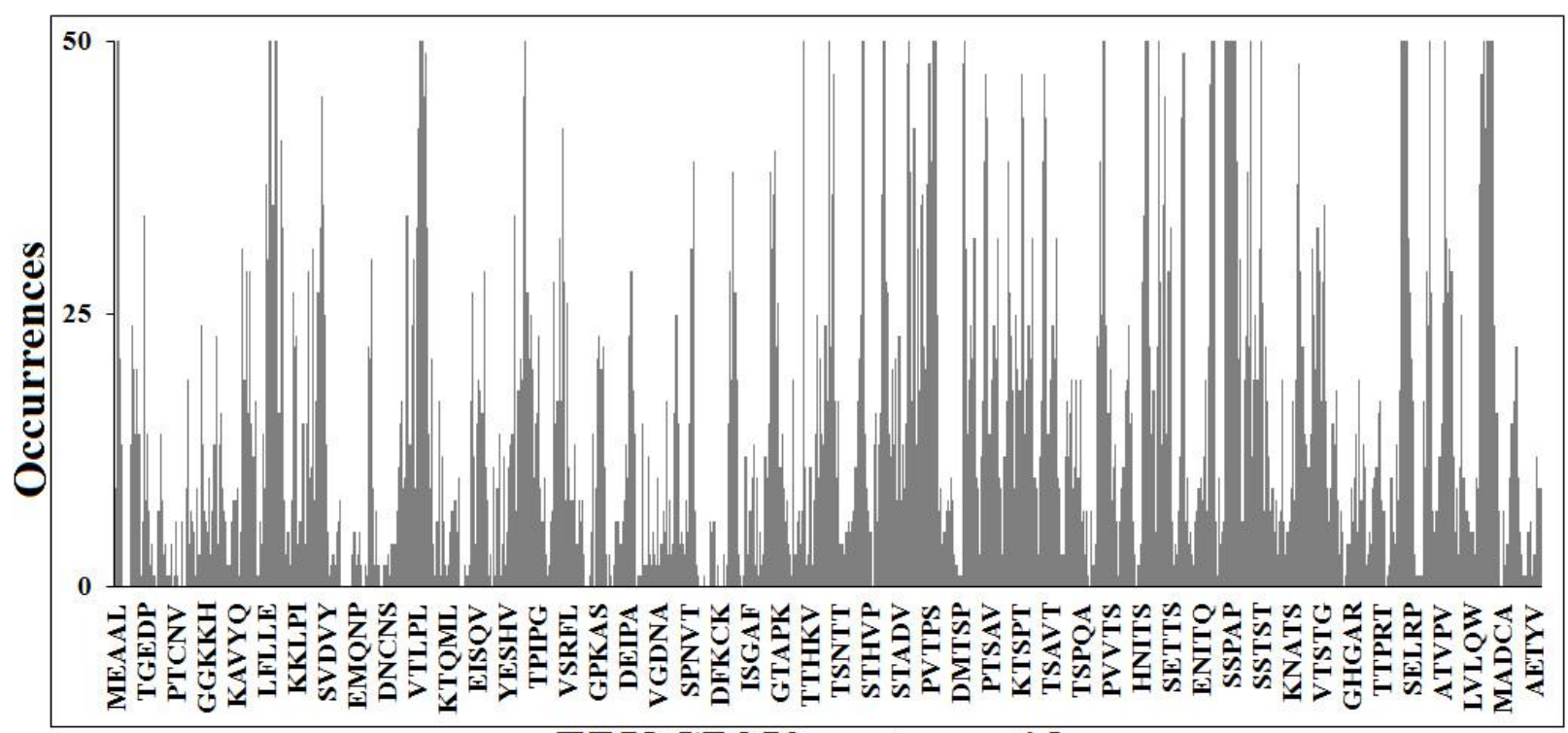

\section{EBV GP350 pentapeptides}

Figure A1. Pentapeptide identity platform shared between EBV GP350 protein and the human proteome. Peptide aa sequences in one letter code.

\section{References}

1. Saha, A.; Robertson, E.S. Epstein-Barr virus-associated B-cell lymphomas: Pathogenesis and clinical outcomes. Clin. Cancer. Res. 2011, 17, 3056-3063.

2. Mueller, N.E.; Grufferman, S. Hodgkin lymphoma. In Cancer Epidemiology and Prevention, 3rd ed; Schottenfeld, D., Fraumeni, J.F., Jr., Eds.; Oxford University Press: New York, NY, USA, 2006; pp. 872-897.

3. Adam, P.; Bonzheim, I.; Fend, F.; Quintanilla-Martínez, L. Epstein-Barr virus-positive diffuse large B-cell lymphomas of the elderly. Adv. Anat. Pathol. 2011, 18, 349-355.

4. Piccaluga, P.P.; Gazzola, A.; Agostinelli, C.; Bacci, F.; Sabattini, E.; Pileri, S.A. Pathobiology of Epstein-Barr virus-driven peripheral T-cell lymphomas. Semin. Diagn. Pathol. 2011, 28, 234244.

5. Huang, W.T.; Lin, C.W. EBV-encoded miR-BART20-5p and miR-BART8 inhibit the IFN- $\gamma-S T A T 1$ pathway associated with disease progression in nasal NK-cell lymphoma. Am. J. Pathol. 2014, 184, 1185-1197.

6. Kanazawa, T.; Hiramatsu, Y.; Iwata, S.; Siddiquey, M.N.; Sato, Y.; Suzuki, M.; Ito, Y.; Goshima, F.; Murata, T.; Kimura, H. Anti-CCR4 monoclonal antibody mogamulizumab for the treatment of EBV-associated T- and NK-Cell lymphoproliferative diseases. Clin. Cancer. Res. 2014, doi:10.1158/1078-0432.CCR-14-0580.

7. Yen, C.Y.; Lu, M.C.; Tzeng, C.C.; Huang, J.Y.; Chang, H.W.; Chen, R.S.; Liu, S.Y.; Liu, S.T.; Shieh, B.; Li, C. Detection of EBV infection and gene expression in oral cancer from patients in Taiwan by microarray analysis. J. Biomed. Biotechnol. 2009, doi:10.1155/2009/904589.

8. Alibek, K.; Kakpenova, A.; Baiken, Y. Role of infectious agents in the carcinogenesis of brain and head and neck cancers. Infect. Agent. Cancer 2013, doi:10.1186/1750-9378-8-7. 
9. Iizasa, H.; Nanbo, A.; Nishikawa, J.; Jinushi, M.; Yoshiyama, H. Epstein-Barr Virus (EBV)-associated Gastric Carcinoma. Viruses 2012, 4, 3420-3439.

10. Incaprera, M.; Rindi, L.; Bazzichi, A.; Garzelli, C. Potential role of the Epstein-Barr virus in systemic lupus erythematosus autoimmunity. Clin. Exp. Rheumatol. 1998, 16, 289-294.

11. Poole, B.D.; Gross, T.; Maier, S.; Harley, J.B.; James, J.A. Lupus-like autoantibody development in rabbits and mice after immunization with EBNA-1 fragments. J. Autoimmun. 2008, 31, 362371.

12. Youinou, P.; Buisson, M.; Berthelot, J.M.; Jamin, C.; Le Goff, P.; Genoulaz, O.; Lamour, A.; Lydyard, P.M.; Seigneurin, J.M. Anti-Epstein-Barr virus-nuclear antigen-1, -2A and -2B antibodies in rheumatoid arthritis patients and their relatives. Autoimmunity 1992, 13, 225-231.

13. Toussirot, E.; Roudier, J. Pathophysiological links between rheumatoid arthritis and the Epstein-Barr virus: An update. Joint Bone Spine 2007, 74, 418-426.

14. Csuka, D.; Simon, D.; Hóbor, R.; Uray, K.; Prohászka, Z.; Bánlaki, Z.; Jani, P.K.; Szilágyi, Á.; Hudecz, F.; Rajczy, K.; et al. Serum concentration of immunoglobulin G-type antibodies against the whole Epstein-Barr nuclear antigen 1 and its aa35-58 or aa398-404 fragments in the sera of patients with systemic lupus erythematosus and multiple sclerosis. Clin. Exp. Immunol. 2013, 171, 255-262.

15. Rand, K.H.; Houck, H.; Denslow, N.D.; Heilman, K.M. Epstein-Barr virus nuclear antigen-1 (EBNA-1) associated oligoclonal bands in patients with multiple sclerosis. J. Neurol. Sci. 2000, $173,32-39$.

16. Hjalgrim, H.; Friborg, J.; Melbye, M. The epidemiology of EBV and its association with malignant disease. In Human Herpesviruses: Biology, Therapy, and Immunoprophylaxis; Arvin, A., Campadelli-Fiume, G., Mocarski, E., Moore, P.S.; Roizman, B., Whitley, R., Yamanishiet, K., Eds.; Cambridge University Press: Cambridge, UK, 2007; Chapter 53.

17. Capone, G.; Calabrò, M.; Lucchese, G.; Fasano, C.; Girardi, B.; Polimeno, L.; Kanduc, D. Peptide matching between Epstein-Barr virus and human proteins. Pathog. Dis. 2013, 69, 205212.

18. Ferlay, J.; Soerjomataram, I.; Ervik, M.; Dikshit, R.; Eser, S.; Mathers, C.; Rebelo, M.; Parkin, D.M.; Forman, D.; Bray. F. GLOBOCAN 2012 v1.0, Cancer Incidence and Mortality Worldwide: IARC CancerBase No. 11 (Internet). Available at: http://globocan.iarc.fr (accessed on 13 December 2013).

19. Browne, P.; Chandraratna, D.; Angood, C.; Tremlett, H.; Baker, C.; Taylor, B.V.; Thompson, A.J. Atlas of multiple sclerosis 2013: A growing global problem with widespread inequity. Neurology 2014, 83, 1022-1024.

20. Uramoto, K.M.; Michet, C.J., Jr.; Thumboo, J.; Sunku, J.; O’Fallon, W.M.; Gabriel, S.E. Trends in the incidence and mortality of systemic lupus erythematosus, 1950-1992. Arthritis Rheum. 1999, 42, 46-50.

21. Gibofsky, A. Overview of epidemiology, pathophysiology, and diagnosis of rheumatoid arthritis. Am. J. Manag. Care 2012, 18, S295-S302.

22. Kanduc, D. Immunogenicity in peptide-immunotherapy: From self/nonself to similar/dissimilar sequences. Adv. Exp. Med. Biol. 2008, 640, 198-207. 
23. Kanduc, D. "Self-nonself" peptides in the design of vaccines. Curr. Pharm. Des. 2009, 15, 32833289.

24. Kanduc, D. Protein information content resides in rare peptide segments. Peptides 2010, 31, 983988.

25. Kanduc, D. The self/nonself issue: A confrontation between proteomes. SelfNonself 2010, 1, 255258.

26. Zeng, M.S.; Li, D.J.; Liu, Q.L.; Song, L.B.; Li, M.Z.; Zhang, R.H.; Yu, X.J.; Wang, H.M.; Ernberg I.; Zeng, Y.X. Genomic sequence analysis of Epstein-Barr virus strain GD1 from a nasopharyngeal carcinoma patient. J. Virol. 2005, 79, 15323-15330.

27. Wu, C.H.; Yeh, L.S.; Huang, H.; Arminski, L.; Castro-Alvear, J.; Chen, Y.; Hu, Z.; Kourtesis, P.; Ledley, R.S.; Suzek, B.E.; et al. The protein information resource. Nucleic Acids Res. 2003, 31, 345-347.

28. Vita, R.; Zarebski, L.; Greenbaum, J.A.; Emami, H.; Hoof, I.; Salimi, N.; Damle, R.; Sette, A.; Peters, B. The immune epitope database 2.0. Nucleic Acids Res. 2010, 38, D854-D862.

29. Thompson, J.D.; Higgins, D.G.; Gibson, T.J. CLUSTAL W: Improving the sensitivity of progressive multiple sequence alignment through sequence weighting, position-specific gap penalties and weight matrix choice. Nucleic Acids Res. 1994, 22, 4673-4680.

30. Benjamin, D.C.; Berzofsky, J.A.; East, I.J.; Gurd, F.R.N.; Hannum, C.; Leach, S.J.; Margoliash, E.; Michael, J.G.; Miller, A.; Prager, E.M.; et al. The antigenic structure of proteins: a reappraisal. Annu. Rev. Immunol. 1984, 2, 67-101.

31. Lerner, R.A. Tapping the immunological repertoire to produce antibodies of predetermined specificity. Nature 1982, doi:10.1038/299592a0.

32. Sutcliffe, J.G.; Shinnick, T.M.; Green, N.; Lerner, R.A. Antibodies that react with predetermined sites on proteins. Science 1983, 219, 660-666.

33. Shinnick, T.M.; Sutcliffe, J.G.; Green, N.; Lerner, R.A. Peptide-elicited protein-reactive antibodies in molecular biology and medicine. J. Invest. Dermatol. 1984, 83, 112S-115S.

34. Niman, H.L.; Houghten, R.A.; Walker, L.E.; Reisfeld, R.A.; Wilson, I.A.; Hogle, J.M.; Lerner, R.A. Generation of protein-reactive antibodies by short peptides is an event of high frequency: Implications for the structural basis of immune recognition. Proc. Natl. Acad. Sci. USA 1983, 80, 4949-4953.

35. Yao, B.; Zhang, L.; Liang, S.; Zhang, C. SVMTriP: A method to predict antigenic epitopes using support vector machine to integrate tri-peptide similarity and propensity. PLOS ONE 2012, 7, e45152.

36. Wang, H.W.; Lin, Y.C.; Pai, T.W.; Chang, H.T. Prediction of B-cell linear epitopes with a combination of support vector machine classification and amino acid propensity identification. J. Biomed. Biotechnol. 2011, doi:10.1155/2011/432830.

37. Singh, H.; Ansari, H.R.; Raghava, G.P.S. Improved method for linear B-cell epitope prediction using antigen's primary sequence. PLOS ONE 2013, 8, e62216.

38. Hemmer, B.; Kondo, T.; Gran, B.; Pinilla, C.; Cortese, I.; Pascal, J.; Tzou, A.; McFarland, H.F.; Houghten, R.; Martin, R. Minimal peptide length requirements for $\mathrm{CD}^{+} \mathrm{T}$ cell clones-implications for molecular mimicry and T cell survival. Int. Immunol. 2000, 12, 375-383.

39. Rothbard, J.B. Peptides and the cellular immune response. Ann. Inst. Pasteur. 1986, 137, 518-526. 
40. Rothbard, J.B.; Taylor, W.R. A sequence pattern common to T cell epitopes. EMBO J. 1988, 7, 93-100.

41. Mathews, J.H.; Allan, J.E.; Roehrig, J.T.; Brubaker, J.R.; Uren, M.F.; Hunt, A.R. T-helper cell and associated antibody response to synthetic peptides of the E glycoprotein of Murray Valley encephalitis virus. J. Virol. 1991, 65, 5141-5148.

42. Reddehase, M.J.; Rothbard, J.B.; Koszinowski, U.H. A pentapeptide as minimal antigenic determinant for MHC class I-restricted T lymphocytes. Nature 1989, 337, 651-653.

43. Geluk, A.; van Meijgaarden, K.E.; Janson, A.A.; Drijfhout, J.W.; Meloen, R.H.; de Vries, R.R.; Ottenhoff, T.H. Functional analysis of DR17(DR3)-restricted mycobacterial T cell epitopes reveals DR17-binding motif and enables the design of allele-specific competitor peptides. J. Immunol. 1992, 149, 2864-2871.

44. Zeng, W.; Pagnon, J.; Jackson, D.C. The C-terminal pentapeptide of LHRH is a dominant B cell epitope with antigenic and biological function. Mol. Immunol. 2007, 44, 3724-3731.

45. Rothbard, J.B.; Pemberton, R.M.; Bodmer, H.C.; Askonas, B.A.; Taylor, W.R. Identification of residues necessary for clonally specific recognition of a cytotoxic T cell determinant. EMBO J. 1989, 8, 2321-2328.

46. Rothbard, J.B.; Gefter, M.L. Interactions between immunogenic peptides and MHC proteins. Annu. Rev. Immunol. 1991, 9, 527-565.

47. Sant'Angelo, D.B.; Robinson, E.; Janeway, C.A., Jr.; Denzin, L.K. Recognition of core and flanking amino acids of MHC class II-bound peptides by the T cell receptor. Eur. J. Immunol. 2002, 32, 2510-2520.

48. Willers, J.; Capone, G.; Lucchese, A. Peptides: An arrival point in cancer vaccinology. Front. Biosci. 2012, 4, 1381-1392.

49. Kanduc, D. Homology, similarity, and identity in peptide epitope immunodefinition. J. Pept. Sci. 2012, 18, 487-494.

50. Kanduc, D. Pentapeptides as minimal functional units in cell biology and immunology. Curr. Protein Pept. Sci. 2013, 14, 111-120.

51. Rowe, M.; Rowe D.T.; Gregory, C.D.; Young, L.S.; Fartell, P.J.; Rupani, H.; Rickinson, A.B. Differences in B cell growth phenotype reflect novel patterns of Epstein-Barr virus latent gene expression in Burkitt's lymphoma cells. EMBO J. 1987, 6, 2743-2751.

52. Sivachandran, N.; Dawson, C.W.; Young, L.S.; Liu, F.F.; Middeldorp, J.; Frappier, L. Contributions of the Epstein-Barr virus EBNA1 protein to gastric carcinoma. J. Virol. 2012, 86, 60-68.

53. Tsang, C.W.; Lin, X.; Gudgeon, N.H.; Taylor, G.S.; Jia, H.; Hui, E.P.; Chan, A.T.; Lin, C.K.; Rickinson, A.B. CD4 ${ }^{+}$T-cell responses to Epstein-Barr virus nuclear antigen EBNA1 in Chinese populations are highly focused on novel C-terminal domain-derived epitopes. J. Virol. 2006, 80, 8263-8266.

54. Long, H.M.; Chagoury, O.L.; Leese, A.M.; Ryan, G.B.; James, E.; Morton, L.T.; Abbott, R.J.; Sabbah, S.; Kwok, W.; Rickinson, A.B. MHC II tetramers visualize human $\mathrm{CD}^{+} \mathrm{T}$ cell responses to Epstein-Barr virus infection and demonstrate atypical kinetics of the nuclear antigen EBNA1 response. J. Exp. Med. 2013, 210, 933-949. 
55. Long, H.M.; Haigh, T.A.; Gudgeon, N.H.; Leen, A.M.; Tsang, C.W.; Brooks, J.; Landais, E.; Houssaint, E.; Lee, S.P.; Rickinson, A.B.; et al. CD4 ${ }^{+}$T-cell responses to Epstein-Barr virus (EBV) latent-cycle antigens and the recognition of EBV-transformed lymphoblastoid cell lines. J. Virol. 2005, 79, 4896-4907.

56. Capone, G.; Fasano, C.; Lucchese, G.; Calabrò, M.; Kanduc D. Department of Biosciences, Biotechnologies and Biopharmaceutics, University of Bari, Bari, Italy. Unpublished data, 2014.

57. Macalma, T.; Otte, J.; Hensler, M.E.; Bockholt, S.M.; Louis, H.A.; Kalff-Suske, M.; Grzeschik, K.H.; von der Ahe, D.; Beckerle, M.C. Molecular characterization of human zyxin. J. Biol. Chem. 1996, 271, 31470-31478.

58. Sheng, Y.; Hong, J.H.; Doherty, R.; Srikumar, T.; Shloush, J.; Avvakumov, G.V.; Walker, J.R.; Xue, S.; Neculai, D.; Wan, J.W.; et al. A human ubiquitin conjugating enzyme (E2)-HECT E3 ligase structure-function screen. Mol. Cell. Proteomics 2012, 11, 329-341.

59. Schütte, J.; Viallet, J.; Nau, M.; Segal, S.; Fedorko, J.; Minna, J. jun-B inhibits and c-fos stimulates the transforming and trans-activating activities of c-jun. Cell 1989, 59, 987-997.

60. Szremska, A.P.; Kenner, L.; Weisz, E.; Ott, R.G.; Passegué, E.; Artwohl, M.; Freissmuth, M.; Stoxreiter, R.; Theussl, H.C.; Parzer, S.B.; et al. JunB inhibits proliferation and transformation in B-lymphoid cells. Blood 2003, 102, 4159-4165.

61. Davis-Smyth, T.; Duncan, R.C.; Zheng, T.; Michelotti, G.; Levens, D. The far upstream element-binding proteins comprise an ancient family of single-strand DNA-binding transactivators. J. Biol. Chem. 1996, 271, 31679-31687.

62. Chari, A.; Golas, M.M.; Klingenhäger, M.; Neuenkirchen, N.; Sander, B.; Englbrecht, C.; Sickmann, A.; Stark, H.; Fischer, U. An assembly chaperone collaborates with the SMNcomplex to generate spliceosomal SnRNPs. Cell 2008, 135, 497-509.

63. Rivkin, E.; Vella, M.J.; Lahita, R.G. A heterogeneous immune response to an SmD-like epitope by SLE patients. J. Autoimmun. 1994, 7, 119-132.

64. Levitskaya, J.; Sharipo, A.; Leonchiks, A.; Ciechanover, A.; Masucci, M.G. Inhibition of ubiquitin/proteasome-dependent protein degradation by the Gly-Ala repeat domain of the Epstein-Barr virus nuclear antigen 1. Proc. Natl. Acad. Sci. USA 1997, 94, 12616-12621.

65. Levitskaya, J.; Coram, M.; Levitsky, V.; Imreh, S.; Steigerwald-Mullen, P.M.; Klein, G.; Kurilla, M.G.; Masucci, M.G. Inhibition of antigen processing by the internal repeat region of the Epstein-Barr virus nuclear antigen-1. Nature 1995, 375, 685-688.

66. Cheng, H.M.; Foong, Y.T.; Sam, C.K.; Prasad, U.; Dillner, J. Epstein-Barr virus nuclear antigen 1 linear epitopes that are reactive with immunoglobulin $\mathrm{A}(\operatorname{Ig} \mathrm{A})$ or $\operatorname{IgG}$ in sera from nasopharyngeal carcinoma patients or from healthy donors. J. Clin. Microbiol. 1991, 29, 2180 2186.

67. Leen, A.; Meij, P.; Redchenko, I.; Middeldorp, J.; Bloemena, E.; Rickinson, A.; Blake, N. Differential immunogenicity of Epstein-Barr virus latent-cycle proteins for human $\mathrm{CD} 4{ }^{+}$T-helper 1 responses. J. Virol. 2001, 75, 8649-8659.

68. Taylor, G.S.; Haigh, T.A.; Gudgeon, N.H.; Phelps, R.J.; Lee, S.P.; Steven, N.M.; Rickinson, A.B. Dual stimulation of Epstein-Barr Virus (EBV)-specific $\mathrm{CD}^{+}-$and $\mathrm{CD} 8^{+}-\mathrm{T}$-cell responses by a chimeric antigen construct: Potential therapeutic vaccine for EBV-positive nasopharyngeal carcinoma. J. Virol. 2004, 78, 768-778. 
69. Woodberry, T.; Suscovich, T.J.; Henry, L.M.; Davis, J.K.; Frahm, N.; Walker, B.D.; Scadden, D.T.; Wang, F.; Brander, C. Differential targeting and shifts in the immunodominance of Epstein-Barr virus-Specific CD8 and CD4 T cell responses during acute and persistent infection. J. Infect. Dis. 2005, 192, 1513-1524.

70. Krüger, S.; Schroers, R.; Rooney, C.M.; Gahn, B.; Chen, S.Y. Identification of a naturally processed HLA-DR-restricted T-helper epitope in Epstein-Barr virus nuclear antigen type 1. J. Immunother. 2003, 26, 212-221.

71. Petersen, J.; Rhodes, G.; Patrick, K.; Roudier, J.; Vaughan, J.H. Human T cell responses to the Epstein-Barr nuclear antigen-1 (EBNA-1) as evaluated by synthetic peptides. Cell Immunol. 1989, 123, 325-333.

72. Petersen, J.; Rhodes, G.; Roudier, J.; Vaughan, J.H. Altered immune response to glycine-rich sequences of Epstein-Barr nuclear antigen-1 in patients with rheumatoid arthritis and systemic lupus erythematosus. Arthritis Rheum. 1990, 33, 993-1000.

73. Taylor, G.S.; Long, H.M.; Haigh, T.A.; Larsen, M.; Brooks, J.; Rickinson, A.B. A role for intercellular antigen transfer in the recognition of EBV-transformed B cell lines by EBV nuclear antigen-specific CD4 ${ }^{+}$T cells. J. Immunol. 2006, 177, 3746-3756.

74. Depil, S.; Moralès, O.; Castelli, F.A.; Delhem, N.; François, V.; Georges, B.; Dufossé, F.; Morschhauser, F.; Hammer, J.; Maillère, B.; et al. Determination of a HLA II promiscuous peptide cocktail as potential vaccine against EBV latency II malignancies. J. Immunother. 2007, 30, 215-226.

75. Moralès, O.; Depil, S.; Mrizak, D.; Martin, N.; Ndour, P.A.; Dufosse, F.; Miroux, C.; Coll, J.; de Launoit, Y.; Auriault, C.; et al. EBV Latency II-derived peptides induce a specific CD4 ${ }^{+}$ cytotoxic T-cell activity and not a $\mathrm{CD}^{+}$regulatory $\mathrm{T}$-cell response. J. Immunother. 2012, 35, 254-266.

76. Voo, K.S.; Peng, G.; Guo, Z.; Fu, T.; Li, Y.; Frappier, L.; Wang, R.F. Functional characterization of EBV-encoded nuclear antigen 1-specific CD4 ${ }^{+}$helper and regulatory $\mathrm{T}$ cells elicited by in vitro peptide stimulation. Cancer Res. 2005, 65, 1577-1586.

77. Smith, C.; Cooper, L.; Burgess, M.; Rist, M.; Webb, N.; Lambley, E.; Tellam, J.; Marlton, P.; Seymour, J.F.; Gandhi, M.; et al. Functional reversion of antigen-specific $\mathrm{CD}^{+} \mathrm{T}$ cells from patients with Hodgkin lymphoma following in vitro stimulation with recombinant polyepitope. J. Immunol. 2006, 177, 4897-4906.

78. Demachi-Okamura, A.; Ito, Y.; Akatsuka, Y.; Tsujimura, K.; Morishima, Y.; Takahashi, T.; Kuzushima, K. Epstein-Barr virus nuclear antigen 1-specific CD4 ${ }^{+} \mathrm{T}$ cells directly kill Epstein-Barr virus-carrying natural killer and T cells. Cancer Sci. 2008, 99, 1633-1642.

79. Sundström, P.; Nyström, M.; Ruuth, K.; Lundgren, E. Antibodies to specific EBNA-1 domains and HLA DRB1*1501 interact as risk factors for multiple sclerosis. J. Neuroimmunol. 2009, 215, 102-107.

80. Gurer, C.; Strowig, T.; Brilot, F.; Pack, M.; Trumpfheller, C.; Arrey, F.; Park, C.G.; Steinman, R.M.; Münz, C. Targeting the nuclear antigen 1 of Epstein-Barr virus to the human endocytic receptor DEC-205 stimulates protective T-cell responses. Blood 2008, 112, 12311239. 
81. Rooney, C.M.; Smith, C.A.; Ng, C.Y.; Loftin, S.; Li, C.; Krance, R.A.; Brenner, M.K.; Heslop, H.E. Use of gene-modified virus-specific $\mathrm{T}$ lymphocytes to control Epstein-Barr-virus-related lymphoproliferation. Lancet 1995, 345, 9-13.

82. Gu, S.Y.; Huang, T.M.; Ruan, L.; Miao, Y.H.; Lu, H.; Chu, C.M.; Motz, M.; Wolf, H. First EBV vaccine trial in humans using recombinant vaccinia virus expressing the major membrane antigen. Dev. Biol. Stand. 1995, 84, 171-177.

83. Moutschen, M.; Léonard, P.; Sokal, E.M.; Smets, F.; Haumont, M.; Mazzu, P.; Bollen, A.; Denamur, F.; Peeters, P.; Dubin, G.; et al. Phase I/II studies to evaluate safety and immunogenicity of a recombinant gp350 Epstein-Barr virus vaccine in healthy adults. Vaccine 2007, 25, 46974705.

84. Sokal, E.M.; Hoppenbrouwers, K.; Vandermeulen, C.; Moutschen, M.; Léonard, P.; Moreels, A.; Haumont, M.; Bollen, A.; Smets, F.; Denis, M. Recombinant gp350 vaccine for infectious mononucleosis: A phase 2, randomized, double-blind, placebo-controlled trial to evaluate the safety, immunogenicity, and efficacy of an Epstein-Barr virus vaccine in healthy young adults. J. Infect. Dis. 2007, 196, 1749-1753.

85. Cui, X.; Cao, Z.; Sen, G.; Chattopadhyay, G.; Fuller, D.H.; Fuller, J.T.; Snapper, D.M.; Snow, A.L.; Mond, J.J.; Snapper, C.M. A novel tetrameric gp350 1-470 as a potential Epstein-Barr virus vaccine. Vaccine 2013, 31, 3039-3045.

86. Ruiss, R.; Jochum, S.; Wanner, G.; Reisbach, G.; Hammerschmidt, W.; Zeidler, R. A virus-like particle-based Epstein-Barr virus vaccine. J. Virol. 2011, 85, 13105-13113.

87. Antsiferova, O.; Müller, A.; Rämer, P.C.; Chijioke, O.; Chatterjee, B.; Raykova, A.; Planas, R.; Sospedra, M.; Shumilov, A.; Tsai, M.H.; et al. Adoptive transfer of EBV specific CD8 ${ }^{+} \mathrm{T}$ sell clones can transiently control EBV infection in humanized mice. PLOS Pathog. 2014, 10, e1004333.

88. Lutzky, V.P.; Crooks, P.; Morrison, L.; Stevens, N.; Davis, J.E.; Corban, M.; Hall, D.; Panizza, B.; Coman, W.B.; Coman, S.; et al. Cytotoxic T cell adoptive immunotherapy as a treatment for nasopharyngeal carcinoma. Clin. Vaccine Immunol. 2014, 21, 256-259.

89. Ghosh, S.K.; Perrine, S.P.; Faller, D.V. Advances in virus-directed therapeutics against EpsteinBarr Virus-associated malignancies. Adv. Virol. 2012, doi:10.1155/2012/509296.

90. Lünemann, J.D.; Tintoré, M.; Messmer, B.; Strowig, T.; Rovira, A.; Perkal, H.; Caballero, E.; Münz, C.; Montalban, X.; Comabella, M. Elevated Epstein-Barr virus-encoded nuclear antigen-1 immune responses predict conversion to multiple sclerosis. Ann. Neurol. 2010, 67, 159-169.

91. Angelini, D.F.; Serafini, B.; Piras, E.; Severa, M.; Coccia, E.M.; Rosicarelli, B.; Ruggieri, S.; Gasperini, C.; Buttari, F.; Centonze, D.; et al. Increased CD8 ${ }^{+} \mathrm{T}$ cell response to Epstein-Barr virus lytic antigens in the active phase of multiple sclerosis. PLOS Pathog. 2013, 9, e1003220.

92. Kannangai, R.; Sachithanandham, J.; Kandathil, A.J.; Ebenezer, D.L.; Danda, D.; Vasuki, Z.; Thomas, N.; Vasan, S.K.; Sridharan, G. Immune responses to Epstein-Barr virus in individuals with systemic and organ specific autoimmune disorders. Indian. J. Med. Microbiol. 2010, 28, 120-123.

93. Mahler, M.; Fritzler, M.J.; Blüthner, M. Identification of a SmD3 epitope with a single symmetrical dimethylation of an arginine residue as a specific target of a subpopulation of anti-Sm antibodies. Arthritis Res. Ther. 2005, 7, R19-R29. 
94. Karin, N.; Binah, O.; Grabie, N.; Mitchell, D.J.; Felzen, B.; Solomon, M.D.; Conlon, P.; Gaur, A.; Ling, N.; Steinman, L. Short peptide-based tolerogens without self-antigenic or pathogenic activity reverse autoimmune disease. J. Immunol. 1998, 160, 5188-5194.

95. Tselios, T.; Daliani, I.; Probert, L.; Deraos, S.; Matsoukas, E.; Roy, S.; Pires, J.; Moore, G.; Matsoukas, J. Treatment of experimental allergic encephalomyelitis (EAE) induced by guinea pig myelin basic protein epitope 72-85 with a human MBP(87-99) analogue and effects of cyclic peptides. Bioorg. Med. Chem. 2000, 8, 1903-1909.

96. Lucchese, A.; Mittelman, A.; Lin, M.S.; Kanduc, D.; Sinha, A.A. Epitope definition by proteomic similarity analysis: Identification of the linear determinant of the anti-Dsg3 MAb 5H10. J. Transl Med. 2004, doi:10.1186/1479-5876-2-43.

97. Lucchese, A.; Mittelman, A.; Tessitore, L.; Serpico, R.; Sinha A.A.; Kanduc D. Proteomic definition of a desmoglein linear determinant common to Pemphigus vulgaris and Pemphigus foliaceous. J. Transl. Med. 2006, doi:10.1186/1479-5876-4-37.

98. Salato, V.K.; Hacker-Foegen, M.K.; Lazarova, Z.; Fairley, J.A.; Lin, M.S. Role of intramolecular epitope spreading in pemphigus vulgaris. Clin Immunol. 2005, 116, 54-64.

99. Angelini, G.; Bonamonte, D.; Lin, M.S., Lucchese, A.; Mittelman, A.; Serpico, R.; Simone, S.; Sinha, A.A.; Kanduc, D. Characterization of polyclonal antibodies raised against a linear peptide determinant of desmoglein-3. J. Exp. Ther. Oncol. 2005, 5, 1-7.

100. Angelini, G.; Bonamonte, D.; Lucchese, A.; Favia, G.; Serpico, R.; Mittelman, A.; Simone, S.; Sinha, A.A.; Kanduc D. Preliminary data on Pemphigus vulgaris treatment by a proteomics-defined peptide: A case report. J. Transl. Med. 2006, doi:10.1186/1479-5876-4-43.

(C) 2015 by the authors; licensee MDPI, Basel, Switzerland. This article is an open access article distributed under the terms and conditions of the Creative Commons Attribution license (http://creativecommons.org/licenses/by/4.0/). 